\title{
Top quark measurements in the CMS experiment
}

\author{
Luca Lista ${ }^{1, a}$ on behalf of the CMS Collaboration \\ ${ }^{1}$ INFN Sezione di Napoli
}

\begin{abstract}
Experimental results on top-quark physics obtained at the CMS experiment are reported based on the data recorded at centre-of-mass energy up to $13 \mathrm{TeV}$. Inclusive and differential cross sections for both top-quark pair and single top-quark production are presented, as well as measurements of top-quark properties in production and decay, and searches for anomalous couplings. The presented measurements test theoretical predictions, including recent perturbative QCD calculations, provide constraints of fundamental standard model parameters, and set limits on physics beyond the standard model.
\end{abstract}

\section{Introduction}

The top quark is the heaviest known elementary particle. It has the largest Yukawa coupling with the Higgs boson:

$$
\kappa_{\mathrm{t}}=\sqrt{2} \frac{m_{\mathrm{t}}(182.5 \mathrm{GeV})}{v(246 \mathrm{GeV})} \simeq 1,
$$

and its lifetime is too short to form bound states $\left(\tau \simeq 0.4 \times 10^{-24} \mathrm{~s}\right)$. The top-quark mass has impact on the stability of the Higgs field:

$$
V(\Phi)=\frac{1}{2} \mu^{2} \Phi^{2}+\frac{1}{4} \lambda(\text { scale })=\Phi^{4}
$$

and gives the largest contribution to pure EWK radiative corrections, being proportional to $G_{\mathrm{F}} m_{\mathrm{t}}^{2}$.

The Large Hadron Collider (LHC) is effectively a a top-quark factory, as can be quantitatively seen in Table 1, where LHC performances are compared with the KEKb B factory. Top quarks can be produced at the LHC via single-top electroweak production or via top-pair strong production, which is the most abundant channel, the major production contribution being gluon fusion, which contributes, at $13 \mathrm{TeV}$, for about $86 \%$ of the total $\mathrm{t}$ production cross section.

\section{Single top-quark production}

Single top quark production has been measured by CMS [1] at energies from 7 to $13 \mathrm{TeV}$ in the $t-$ and $s$ - channel and in the tW associated mode.

The most recent measurement [2] of single top production cross section at $14 \mathrm{TeV}$ gives:

$$
\sigma_{t-\text { ch. }}=238 \pm 13 \text { (stat) } \pm 29 \text { (syst) pb , }
$$

\footnotetext{
ae-mail: luca.lista@na.infn.it
} 
Table 1. Comparison of KEKb design luminosity and $\Upsilon(4 S)$ production cross section with LHC peak luminosity and top-pair production cross section.

\begin{tabular}{lll}
\hline & KEKb & LHC \\
\hline quark type & $\mathrm{b}$ & $\mathrm{t}$ \\
\hline$\sigma(\mathrm{nb})$ & $1.15(\Upsilon(4 S))$ & $0.83(\mathrm{tt}$, incl. $)$ \\
$L\left(\mathrm{~cm}^{-2} \mathrm{~s}^{-1}\right)$ & $2.1 \times 10^{34}$ & $1.74 \times 10^{34}$ \\
\hline
\end{tabular}

and the ratio of top to antitop production cross setion is measured to be:

$$
R_{t-\text { ch. }}=\frac{\sigma_{\mathrm{t}}}{\sigma_{\overline{\mathrm{t}}}}=1.81 \pm 0.18(\text { stat }) \pm 0.15(\text { syst })
$$

and is consistent with the asymmetry expected from the parton distribution functions (PDFs) of the proton. The precision in the measurement of $R_{t-\mathrm{ch}}$ does not yet allow to set further constrints on proton PDF. From single top production cross section, the CKM matrix element $\left|V_{\mathrm{tb}}\right|$ can be determined, accounting for a possible presence of an anomalous Wtb coupling, which is taken into account with the form factor $f_{\mathrm{LV}}$ :

$$
\left|f_{\mathrm{LV}} V_{\mathrm{tb}}\right|=1.05 \pm 0.07(\exp ) \pm 0.02(\mathrm{th})
$$

All measurements agree so far with the Standard Model (SM) predictions.

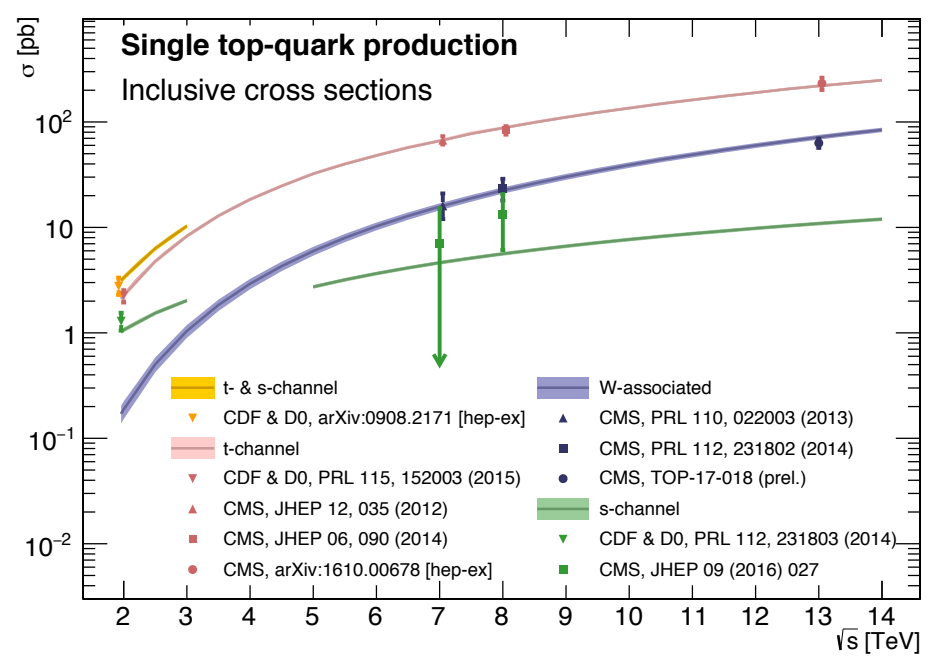

Figure 1. Summary of single top cross section measurements by CMS, as function of centre-of-mass energy. This plot is from Ref. [3].

A summary of single-top cross section measurements at different center-of-mass energies and in different channels by CMS is visible in Fig. 1. 


\section{3 ț production}

The $t \bar{t}$ production has been measured at different center-of-mass energy and exploiting different topquark decay channels. One of the most recent measurements is in the $\ell+$ jets channel at $13 \mathrm{TeV}$ [4], which gives the following cross section:

$$
\sigma=888 \pm 2(\text { stat })_{-28}^{+26}(\text { syst }) \pm 20(\text { lumi }) \mathrm{pb},
$$

in agreement with the SM predictions.

The dependence of $\bar{t}$ production cross section on the top quark pole mass allows to determine, indirectly:

$$
m_{\mathrm{t}}(\text { pole })=170.6 \pm 2.7 \mathrm{GeV},
$$

which, though with less precision, is complementary to the direct mass measurements (see Sect. 4).

A dedicated run at a reduced center-of-mass energy of $5 \mathrm{TeV}$ in November 2015 allowed to measure the $\bar{t} \bar{t}$ production cross section at an energy intermediate between Tevatron and LHC [5]. At lower energy the contribution of gluon fusion to the total production cross section lowers from $86 \%$ at 13 $\mathrm{TeV}$ to $73 \%$, allowing to set new constraints on the gluon PDFs, though with still a limitated precision due to the small collected integrated luminosity of $27.4 \mathrm{pb}^{-1}$.

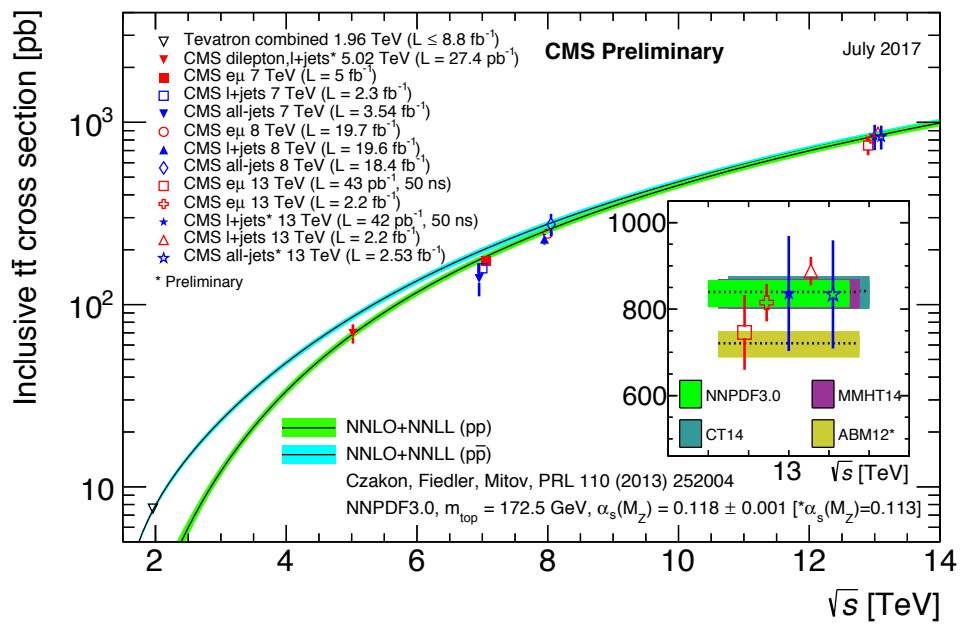

Figure 2. Top quark pair cross section summary of most precise CMS measurements in the dilepton and $\ell+$ jets channel in comparison with the theory calculation at NNLO+NNLL accuracy. The Tevatron measurements are also shown. This plot is from Ref. [3].

A summary of the measured top-pair cross sections at different center-of-mass by CMS is visible in Fig. 2.

$\mathrm{t} \overline{\mathrm{t}}$ production has been studied in details with measurements of differential cross sections [6] as a function of different kinematic variables with definition of the top quark at particle level. Measurements have been compared with SM prediction computed at the Next-to-leading order in QCD showing a good agreement with the theory.

In general, all $t \bar{t}$ measurements performed at different center-of-mass energies show good agreement with theory and allow to set constraints on the proton PDFs. 


\section{Top quark mass measurements}

The top quark mass is an important parameter in the SM, entering radiative corrections and affecting the stability of the Higgs potential.

CMS has measured $m_{\mathrm{t}}$ in different channels using different methods. The most precise measurement exploits the $\mu+$ jets channel at $13 \mathrm{TeV}$ reconstructing the top mass with a kinematic fit that imposes the constaint of the $\mathrm{W}$ mass in the top decay products. A requirement on the goodness-of-fit reduces spurious combinations. The top mass is fit together with a possible residual jet-energy scale factor (JSF) in order to reduce systematic uncertainties due to jet-energy calibrations. The result is [7]:

$$
m_{\mathrm{t}}=172.62 \pm 0.38(\text { stat }+\mathrm{JSF}) \pm 0.70(\text { syst }) \mathrm{GeV} \text {. }
$$

Alternative approaches are also adopted for top mass measurements, in order to provide complementary systematic uncertainties. One recent example is the measurement of $m_{\mathrm{t}}$ using spectra of kinematical variables, such as the invariant mass of the lepton and b quark from the top quark decay, or the transverse invariant mass of the b-quark pair from the decay of the two top quarks [8], which exhibit a kinematic endpoint sensitive to $m_{\mathrm{t}}$. The measured top-quark mass value, combined with different alternative approaches, is [9]:

$$
m_{\mathrm{t}}=172.50 \pm 0.21(\text { stat }) \pm 0.72 \text { (syst) } \mathrm{GeV} .
$$

The result is in good agreement with the combination of 'standard' techniques [10]:

$$
m_{\mathrm{t}}=172.43 \pm 0.13 \text { (stat) } \pm 0.46(\text { syst }) \mathrm{GeV},
$$

but the achieved precision in the alternative procedures is not sufficient yet to improve the overall combination, once combined with the 'standard' measurements.

The top-quark mass has also been measured in highly boosted events where the invariant mass spectrum has been used [11] for jets reconstructed with a wide cone radius parameter $(R=1.2)$, aiming at the reconstruction of the decay product of a top quark into a single 'merged' jet. The uncertainty in the top quark mass measurement is so far limited $( \pm 9 \mathrm{GeV})$, dominated by statistical uncertainty and jet energy scale uncertainty in the boosted regime. Potential improvements and more constraints in systematic uncertainties are anyway possible in future application of this method on largar data samples.

Figure 3 shows a summary of CMS $m_{\mathrm{t}}$ measurements done during Run-I.

\section{Top quark mass width}

The width of the top quark is predicted in the SM to be $1.35 \mathrm{GeV}$. The invariant mass spectrum of the lepton and jet has been considered to measure the top width [13], including boosted jet reconstruction, leading to the following confidence interval, determined at the $95 \%$ confidence level (CL):

$$
0.6 \leq \Gamma_{\mathrm{t}} \leq 2.5 \mathrm{GeV}
$$

This measurement compares with the measurement of top width from indirect methods [14], which has about a $10 \%$ uncertainty. 


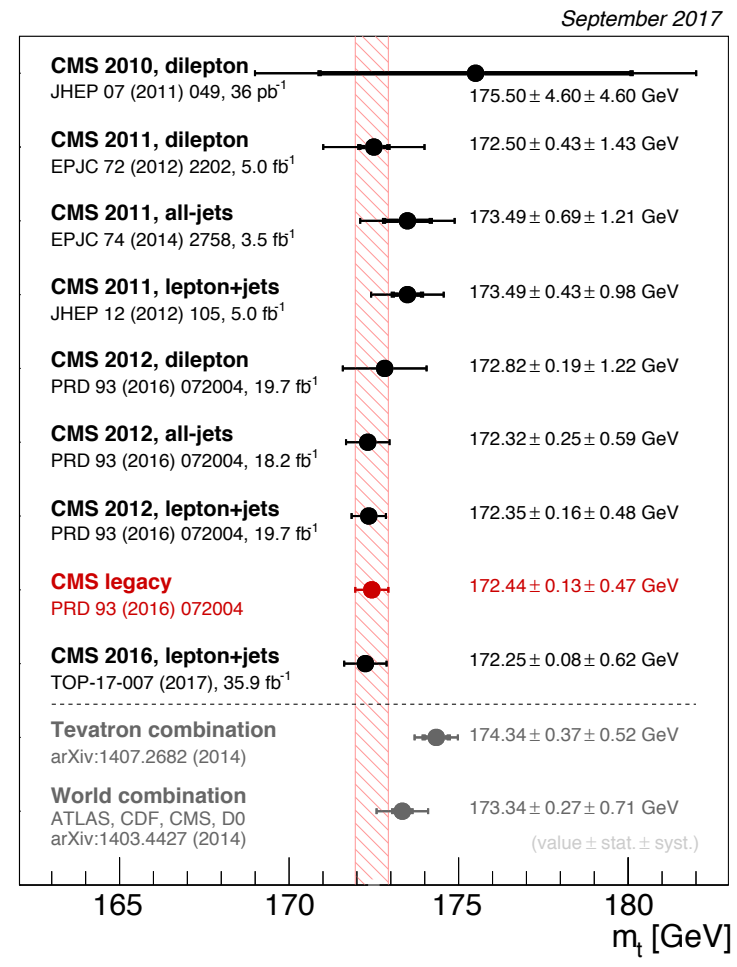

Figure 3. Summary of Run-I CMS $m_{\mathrm{t}}$ measurements and their combination. This plot is from Ref. [12].

\section{Top pair production in association with vector bosons}

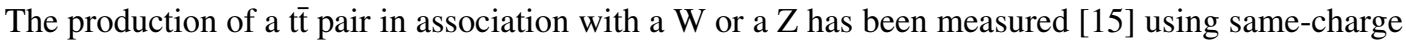
dilepton event with three- and four-lepton final states. Jet and b-jet multiplicities have been used to enhance the signal-to-background ratio. The following cross sections have been determined:

$$
\begin{aligned}
\sigma(\mathrm{pp} \rightarrow \mathrm{t} \overline{\mathrm{t} Z}) & =1.00_{-0.08}^{+0.09}(\text { stat })_{-0.10}^{+0.12} \text { (syst) } \mathrm{pb}, \\
\sigma(\mathrm{pp} \rightarrow \mathrm{t} \overline{\mathrm{t}}) & =0.80_{-0.11}^{+0.12}(\text { stat })_{-0.12}^{+0.13}(\text { syst }) \mathrm{pb}, \\
\sigma\left(\mathrm{pp} \rightarrow \mathrm{t} \mathrm{t}^{+}\right) & =0.58 \pm 0.09(\text { stat })_{-0.08}^{+0.09}(\text { syst }) \mathrm{pb}, \\
\sigma\left(\mathrm{pp} \rightarrow \mathrm{t} \mathrm{tW}^{-}\right) & =0.19 \pm 0.07 \text { (stat) } \pm 0.06 \text { (syst) } \mathrm{pb} .
\end{aligned}
$$

Constraints are set on Wilson coefficients of operators for $\mathrm{ttZ}$ and $\mathrm{ttW}$ production predicted by new physics models beyond the SM (BSM).

The production cross section of a top quark pair in association with a photon has been measured [16] for semileptonic events in the fiducial volume defined applying the following requirement to the final-state particles:

- e: $p_{\mathrm{T}}>35 \mathrm{GeV},|\eta|<2.5$, 
- $\mu: p_{\mathrm{T}}>26 \mathrm{GeV},|\eta|<2.4$,

- $\gamma: p_{\mathrm{T}}>25 \mathrm{GeV},|\eta|<1.44$,

- $v: p_{\mathrm{T}}$ sum of the two neutrinos $>20 \mathrm{GeV}$.

Photon isolation is used to discriminate non-prompt photons. The masured fiducial cross section, combining the electron and muon channels, relative to the top pair production cross section, is:

$$
R=\sigma_{\mathrm{t} \bar{\gamma}}^{\mathrm{fid}} / \sigma_{\mathrm{tt}}=(5.2 \pm 1.1) \times 10^{-4},
$$

and the cross section, times the considered decay branching fraction is:

$$
\sigma_{\mathrm{t} \bar{\gamma}} \times \mathcal{B}=515 \pm 108 \mathrm{fb}
$$

in agreement with the SM prediction of $591 \pm 71$ (scale) $\pm 30(\mathrm{PDF}) \mathrm{fb}$.

\section{Single top and flavour-changing neutral current}

Events with a $\mathrm{Z}$ boson accompanying a single top quark allow to study anomalous flavour-changing neutral currents foreseen in BSM models [17] whose couplings can be modeled using effective operators. Searches for a BSM signal have been done discriminating SM backgrounds using boosted decision trees (BDT) multivariate discriminators in events with three leptons in the final state. The observed yield is compatible with SM in different signal/control regions, and the following cross section has been measured:

$$
\sigma\left(\mathrm{pp} \rightarrow \mathrm{tZq} \rightarrow \ell v \mathrm{~b} \ell^{+} \ell^{-} \mathrm{q}\right)=10_{-7}^{+8} \mathrm{fb}
$$

to be compared with the SM prediction of $8.2 \mathrm{fb}$. The following limits have been determined for the FCNC transition branching fractions at the $95 \%$ confidence level (CL):

$$
\begin{aligned}
& \mathcal{B}(\mathrm{t} \rightarrow \mathrm{Zu})<0.022 \% \\
& \mathcal{B}(\mathrm{t} \rightarrow \mathrm{Zc})<0.049 \%
\end{aligned}
$$

BSM models predict FCNC branching fractions up to $\sim 10^{-4} \div 10^{-3}$.

\section{Four top production}

An example of a process that is very rare in the SM, but may be significantly enhanced in BSM models, is the production of four top quarks. The SM production cross section prediction is only 1.3 $\mathrm{fb}$. Single lepton and opposite-sign dilepton + jets events have been studied with an extensive usage of BDT techniques [18], combining global event and jet properties. A first BDT ranks 3-jet combinations consistent with all-hadronic top quark decays based on invariant masses and b-tag information; then, a second BDT uses the ranking from the first BDT plus global event kinematic variables to identify signal events. The upper limit to four-top production cross section is, at the 95\% CL:

$$
\sigma_{\mathrm{ttt \textrm {t }}}<94 \mathrm{fb}=10.2 \times \mathrm{SM} .
$$

Combined with the same-sign dilepton channel, the limit improves to:

$$
\sigma_{\text {ttitt }}<69 \mathrm{fb}=7.4 \times \mathrm{SM} .
$$




\section{Future perspectives}

Long-term perspectives studies were prepared for the 2014 Snowmass meeting [19] and for ECFA 2016 [20] based on extrapolations of the LHC integratedluminosity and potential analysis technique improvements.

Prospects for Yukawa coupling measured with ttH Higgs boson production, studied in different final states, give the following possible precision levels:

$$
\begin{aligned}
& \kappa_{\mathrm{t}} \rightarrow 14 \div 15 \%\left(300 \mathrm{fb}^{-1}\right), \\
& \kappa_{\mathrm{t}} \rightarrow 7 \div 10 \%\left(3 \mathrm{ab}^{-1}\right) .
\end{aligned}
$$

Expectations for FCNC, still based on old preliminary results at $8 \mathrm{TeV}$, give the following potential upper limits:

$$
\begin{aligned}
\mathcal{B}(\mathrm{t} \rightarrow \mathrm{Zu}, \mathrm{c}) & <1 \times 10^{-4}\left(3 \mathrm{ab}^{-1}\right) \\
\mathcal{B}(\mathrm{t} \rightarrow \gamma \mathrm{u}) & <2.6 \times 10^{-4}\left(3 \mathrm{ab}^{-1}\right)
\end{aligned}
$$

The top-quark mass may be measured with a precision down to about $200 \mathrm{MeV}$, exploiting a combination of standard and alternative techniques.

\section{Conclusions}

Many precision measurements have been performed by CMS at $13 \mathrm{TeV}$ and more are ongoing after legacy measurements were established at 7 and $8 \mathrm{TeV}$. No deviation from the Standard Model have been observed so far, but the search continues with more and more precision exploiting the high luminosity of future LHC runs. More channels are opening with higher luminosity, with the possibility to probe possible deviations from the Standard Model.

\section{References}

[1] CMS Collaboration, The CMS experiment at the CERN LHC, JINST 3 S08004 (2008).

[2] CMS Collaboration, Cross section measurement of t-channel single top quark production in pp collisions at $\sqrt{s}=13 \mathrm{TeV}$, CMS-TOP-16-003, arXiv:1610.00678, submitted to Phys. Lett. B. (2017).

[3] CMS Collaboration, Top Quark Physics Summary Figures: https://twiki.cern.ch/twiki/bin/view/CMSPublic/PhysicsResultsTOPSummaryFigures

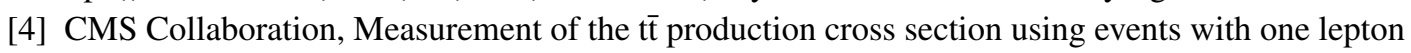
and at least one jet in pp collisions at $\sqrt{s}=13 \mathrm{TeV}$, CMS-TOP-16-006, arXiv:1701.06228, J. High Energy Phys. 09051 (2017).

[5] CMS Collaboration, Measurement of the inclusive $t \bar{t}$ at $\sqrt{s}=5.02 \mathrm{TeV}$, CMS-PAS-TOP-16-023 (2017). https://cds.cern.ch/record/2258177/

[6] CMS Collaboration, Measurement of normalized differential tt cross sections in the dilepton channel from pp collisions at $\sqrt{s}=13 \mathrm{TeV}$, CMS-TOP-16-007, arXiv:1708.07638, submitted to J. High Energy Phys. (2017).

[7] CMS Collaboration, Measurement of the top quark mass with muon+jets final states in pp collisions at $\sqrt{s}=13 \mathrm{TeV}$, CMS-PAS-TOP-16-022 (2017). http://cds.cern.ch/record/2255834

[8] CMS Collaboration, Measurement of the top quark mass in the dileptonic $t \bar{t}$ decay channel using the mass observables $M_{\mathrm{b} \ell}, M_{\mathrm{T} 2}$, and $M_{\mathrm{b} \ell v}$ in pp collisions at $\sqrt{s}=8 \mathrm{TeV}$, CMS-TOP-15-008, arXiv:1704.06142, Phys. Rev. D 96, 032002 (2017). 
[9] CMS Collaboration, Combinations of the CMS alternative technique measurements of the top quark mass, CMS-PAS-TOP-15-012 (2016). https://cds.cern.ch/record/2235162

[10] CMS Collaboration, Measurement of the top quark mass using proton-proton data at $\sqrt{s}=7$ and 8 TeV, CMS-TOP-14-022, arXiv:1509.04044, Phys. Rev. D 93, 072004 (2016).

[11] CMS Collaboration, Measurement of the jet mass in highly boosted $\bar{t} \bar{t}$ events from pp collisions at $\sqrt{s}=8 \mathrm{TeV}$, CMS-TOP-15-015, arXiv:1703.06330, Eur. Phys. J. C 77467 (2017).

[12] CMS Collaboration, Measurement of the top quark mass using proton-proton data at $\sqrt{s}=7$ and 8 TeV, CMS TOP-14-022, arXiv:1509.04044, Phys. Rev. D 93, 072004 (2016).

[13] CMS Collaboration, Bounding the top quark width using final states with two charged leptons and two jets at $\sqrt{s}=13 \mathrm{TeV}$, CMS-PAS-TOP-16-019 (2016). https://cds.cern.ch/record/2218019

[14] CMS Collaboration, Measurement of the ratio $\mathcal{B}(\mathrm{t} \rightarrow \mathrm{Wb}) / \mathcal{B}(\mathrm{t} \rightarrow \mathrm{Wq})$ in pp collisions at $\sqrt{s}=$ 8 TeV, CMS-TOP-12-035, arXiv:1404.2292, Phys. Lett. B 73633 (2014).

[15] CMS Collaboration, Measurement of top pair-production in association with a $\mathrm{W}$ or $\mathrm{Z}$ boson in pp collisions at $13 \mathrm{TeV}$, CMS-PAS-TOP-17-005 (2017). https://cds.cern.ch/record/2264544

[16] CMS Collaboration, Measurement of the semileptonic $\overline{\mathrm{t}} \gamma$ production cross section in pp collisions at $\sqrt{s}=8 \mathrm{TeV}$, CMS-TOP-14-008, arXiv:1706.08128, submitted to J. High Energy Phys. (2017).

[17] CMS Collaboration, Search for associated production of a $\mathrm{Z}$ boson with a single top quark and for $\mathrm{tZ}$ flavour-changing interactions in pp collisions at $\sqrt{s}=8 \mathrm{TeV}$, CMS-TOP-12-039, arXiv:1702.01404, J. High Energy Phys 07003 (2017).

[18] CMS Collaboration, Search for standard model production of four top quarks in proton-proton collisions at $\sqrt{s}=13$ TeV, CMS-TOP-16-016, arXiv:1702.06164, Phys. Lett. B 772336 (2017).

[19] K. Agashe et al., Snowmass 2013 Top quark working group report, arXiv:1311.2028 (2013).

[20] CMS Collaboration, ECFA 2016: Prospects for selected standard model measurements with the CMS experiment at the High-Luminosity LHC, CMS-PAS-FTR-16-006 (2016). https://cds.cern.ch/record/2262606 